\title{
Source-region characteristics of anemone active regions in the ascending phase of solar cycle 24
}

\author{
R. Sharma and C. Cid

\begin{abstract}
Space Weather Research Group, Departamento de Física y Matemáticas, Universidad de Alcalá, A-2 km 33,600, 28871 Alcalá de Henares, Madrid, Spain

e-mail: rahul.sharma@uah.es, consuelo.cid@uah.es
\end{abstract}

Received 14 July 2020 / Accepted 19 August 2020

\begin{abstract}
Context. Active regions in close proximity to coronal holes, also known as anemone regions, are the best candidates for studying the interaction between closed and open magnetic field topologies at the Sun. Statistical investigation of their source-region characteristics can provide vital clues regarding their possible association with energetic events, relevant from space weather perspectives.

Aims. The main goal of our study is to understand the distinct properties of flaring and non-flaring anemone active regions and their host coronal holes, by examining spatial and magnetic field distributions during the rise phase of the solar cycle, in the years 20112014.

Methods. Anemone regions were identified from the minimum-distance threshold, estimated using the data available in the online catalogs for on-disk active regions and coronal holes. Along with the source-region area and magnetic field characteristics, associated filament and flare cases were also located. Regions with and without flare events were further selected for a detailed statistical examination to understand the major properties of the energetic events, both eruptive and confined, at the anemone-type active regions. Results. Identified anemone regions showed weak asymmetry in their spatial distribution over the solar disk, with yearly average independent from mean sunspot number trend, during the rise phase of solar cycle 24. With the progression in solar cycle, the area and minimum-distance parameters indicated a decreasing trend in their magnitudes, while the magnetic field characteristics indicated an increase in their estimated magnitudes. More than half of the regions in our database had an association with a filament structure, and nearly a third were linked with a magnetic reconnection (flare) event. Anemone regions with and without flares had clear distinctions in their source-region characteristics evident from the distribution of their properties and density analysis. The key differences included larger area and magnetic field magnitudes for flaring anemone regions, along with smaller distances between the centers of the active region and its host coronal hole.
\end{abstract}

Key words. Sun: activity - Sun: magnetic fields - Sun: flares - solar-terrestrial relations

\section{Introduction}

Magnetic field topology plays an important factor in triggering the solar eruptive events and in conditioning the geomagnetic disturbances in near-Earth space environment. At the Sun, openand closed-field magnetic configurations outline the observed flux features and associated complexities, that serve as a critical element for the reconnection process (Priest \& Forbes 2000). Coronal holes (CHs) are large-scale manifestations of open magnetic field lines in solar corona (Cranmer 2009) and appear relatively dark and cold when observed in extreme ultraviolet (EUV) and x-ray emissions. These regions appear dark (and cold) due to depletion of plasma through open field lines, resulting in lowered plasma density (and temperature) compared to the ambient corona. The escaped plasma, along with open field magnetic structure is associated with corotating high-speed streams, which are sources of high-velocity solar wind and moderate geomagnetic activity at the Earth (Grandin et al. 2019).

On the other hand, active regions (ARs) reflect the coronal extension of low-lying photospheric magnetic field concentrations. These closed-field topology features are key sites of all major large-scale magnetic activity on the Sun, such as flux emergence, cancellation, and helicity (see review by Toriumi \& Wang 2019). The available magnetic flux densities, along with the complexity and/or variability of the flux features at these regions are closely linked with the observed explosive events on the Sun. Moreover, these coronal features are routinely associated with huge expulsions of plasma and magnetic fields in the interplanetary medium, also known as coronal mass ejections (CMEs). Interplanetary counterparts of CME events are predominantly responsible for intense geomagnetic disturbances in near-Earth space environment (Gopalswamy 2006; Kilpua et al. 2017).

However, EUV and x-ray observations also revealed ubiquitous features with closed-field topology within or surrounded by open magnetic field regions. This distinct class of ARs were first noted as "fountain-like" features by Tousey et al. (1973); however, they were later studied and reported as "anemone" regions by Shibata et al. (1994). These active regions are bordered by coronal hole structures and the boundary between openand closed-field configurations, represent preferable sites for the occurrence of interchange reconnection (Crooker \& Owens 2012). This mechanism is key to many observed phenomena associated with the anemone regions such as x-ray jets and the origin of slow solar wind from ARs and CME eruptions. From these x-ray and coronal jets, which originate from the interaction between emerging magnetic flux and open magnetic fields, are most commonly reported small-scale features 

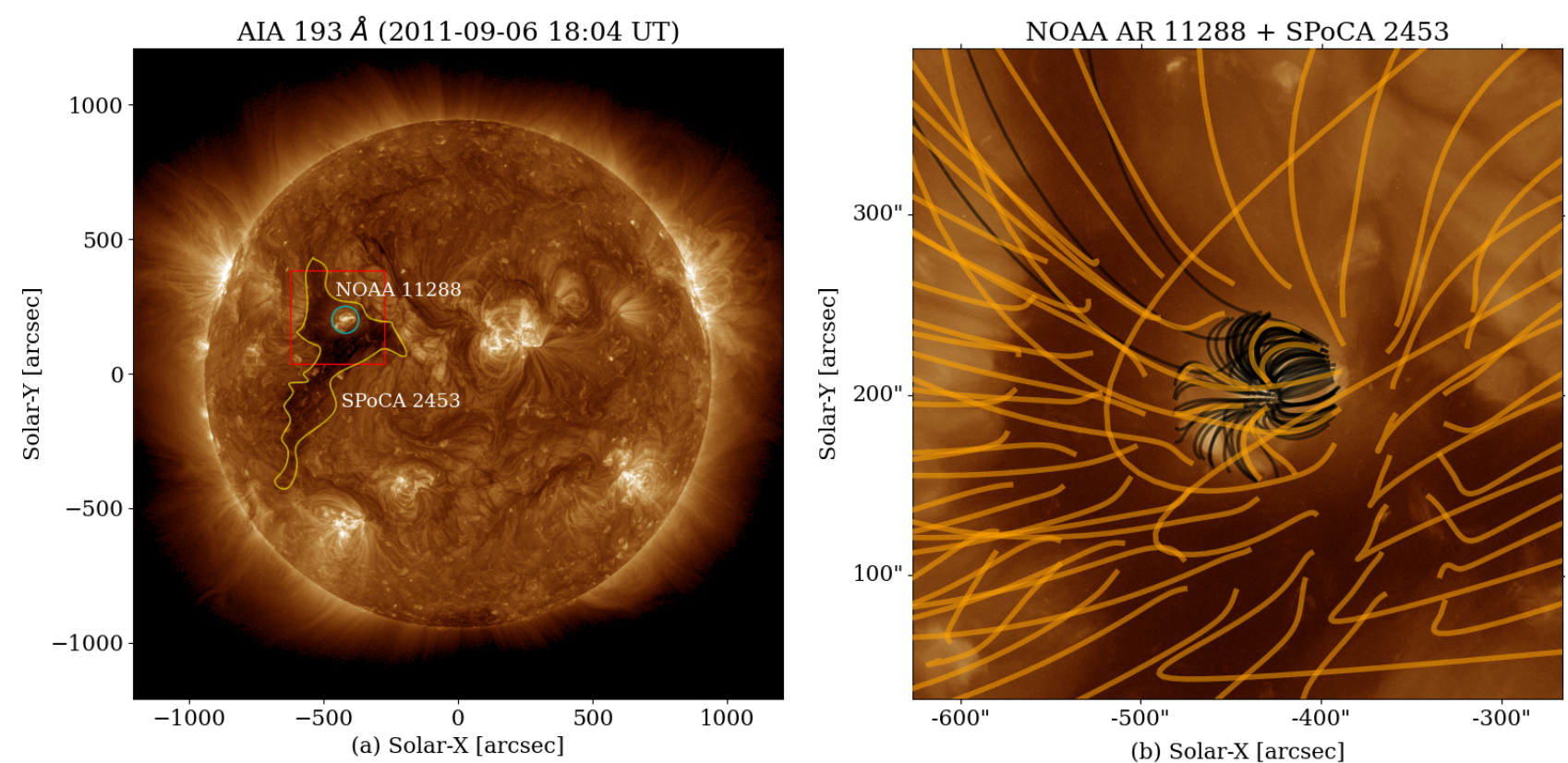

Fig. 1. Example of an anemone region located at the inside edge of a coronal hole feature. Panel $a$ : SDO/AIA $193 \AA$ Amage taken on 06 September 2011, with an anemone active region (NOAA 11288, circle) confined within the coronal hole (SPoCA 2453) boundary, highlighted by a yellow line. The area enclosed in the square (red) is used to estimate the magnetic field topology of the region using the Potential Field Source Surface (PFSS) model, as shown in panel $b$. Extrapolated magnetic field lines for the active region (black) indicate a closed-field topology, resembling a fountain or a sea-anemone feature, surrounded by an open magnetic field (orange) structure from the coronal hole.

(see, e.g, Shibata et al. 2007; Nishizuka et al. 2011; Singh et al. 2011; Moreno-Insertis \& Galsgaard 2013; Kumar et al. 2019) that emanate within the $\mathrm{CH}$ boundary.

From the space weather perspective, anemone regions have received much interest for their potential role in modulating the geoeffectiveness of associated eruptive events. This is primarily due to the presence of open field lines in the vicinity of AR that can influence the propagation of CMEs in the interplanetary medium with high-speed streams, and/or reconnection at the Earth's magnetosphere. Bravo et al. (1998) reported cases with intense geomagnetic storms and highlighted their relationship with corresponding active regions adjacent to the coronal holes, while Chertok et al. (2002) reported filament eruptions associated with the anemone regions. Furthermore, these features were also found to be linked with the disappearance of solar wind at $1 \mathrm{AU}$ (Janardhan et al. 2008), and generation of (slow) solar wind (He et al. 2010; Macneil et al. 2019) due to interaction between closed- and open-field lines in the proximity of anemone regions.

Asai et al. (2008) investigated the magnetic field characteristics of anemone regions, identified in $\mathrm{x}$-ray emission data from the Yohkoh spacecraft. They concluded that anemone regions had much simpler magnetic field configurations compared to other ARs located elsewhere on the Sun. Furthermore, the interplanetary and geomagnetic consequences of an eruptive event associated with an anemone region, with moderate geomagnetic effects on near-Earth space environment, was also reported by Asai et al. (2009). More extensive investigation(s) of near-Sun and interplanetary interactions between CMEs and $\mathrm{CH}$ structures indicated deflection of interplanetary CMEs from the Sun-Earth line (Gopalswamy et al. 2009; Mäkelä et al. 2013; Shugay et al. 2018; Heinemann et al. 2019a). These studies also indicated that any interactions between interplanetary CME structures with fast streams from CHs can strongly influence the arrival times and/or the geomagnetic severity of solar eruptive events.
In this paper, we examine the source-region characteristics of anemone-type active regions, which are located in the periphery of coronal holes. The key physical properties relevant for space weather studies, such as AR-CH distance, area, magnetic field magnitudes for observed features, and their ratio(s) are investigated, along with any possible association with filament and/or flare events. In the following sections, catalogs and datasets used in the study are described (Sect. 2), with the results and discussion of statistical analysis in Sect. 3. The main conclusions are listed in Sect. 4, while the identified anemone-type regions used in the analysis are given in Appendix A.

\section{Database}

The anemone active regions for our investigation were identified by estimating the minimum distance between the active regions and corresponding coronal holes, listed in the available catalogs from the Solar and Space Weather Network of Excellence (SOLSPANET ${ }^{1}$ ), which detects $\mathrm{CH}$ using the spatial possibilistic clustering algorithm (SPoCA) and the Heliophysics Feature Catalogue $\left(\mathrm{HFC}^{2}\right)$. Figure 1a shows an example of one such anemone active region identified for our analysis. The region (NOAA 11288) was observed on 06 September 2011 at 18:04 UT at EUV $193 \AA$ wavelength, from Atmospheric Imaging Assembly (AIA: Lemen et al. 2012) on board the Solar Dynamics Observatory (SDO: Pesnell et al. 2012) spacecraft. The feature appeared to be surrounded by a transequatorial $\mathrm{CH}$ region, observed as a relatively dark feature in EUV emission data.

Topologically, this anemone region which was located near the northern edge of the CH (SPoCA 2453) boundary, had a null-point magnetic field structure (Fig. 1b), evident from the

\footnotetext{
1 http://www. solspanet.eu

2 http://voparis-helio.obspm.fr/hfc-gui/index.php
} 
Potential Field Source Surface (PFSS: Schrijver \& De Rosa 2003) extrapolation of co-spatial magnetogram data. Magnetic configuration of this active region resembled closely a fountainlike and/or sea-anemone appearance, as suggested by previous studies (Tousey et al. 1973; Shibata et al. 1994). Open magnetic field lines from the $\mathrm{CH}$ surrounding the feature can facilitate conditions, ideal for mechanisms like interchange-reconnection and/or the origin of slow solar wind from the active region. For the respective catalogs used in our analysis, the procedures involved in the identification and characterization of spatial properties of on-disk active regions and coronal hole features are provided in detail by Barra et al. (2009), Bagashvili et al. (2017), and Heinemann et al. (2019b). Anemone-type active regions from these catalogs for our investigation were selected as follows.

The location of features in the Helioprojective Cartesian (HPC) coordinate system were taken from the available catalogs, for the center of gravity $(\mathrm{COG})$ of active region $\left(x_{\mathrm{AR}}, y_{\mathrm{AR}}\right)$ and coronal hole $\left(x_{\mathrm{CH}}, y_{\mathrm{CH}}\right)$ structures. The distance between each active region and coronal hole was then estimated as $D_{(\mathrm{CH}-\mathrm{AR})}=$ $\sqrt{\left(x_{\mathrm{AR}}-x_{\mathrm{CH}}\right)^{2}+\left(y_{\mathrm{AR}}-y_{\mathrm{CH}}\right)^{2}}$. This provided a list of AR-CH pairs, along with their corresponding distances for each day of the year. For any given year, the AR-CH pairs were further sorted in bins of equal size, so that the distance parameter $\left(D_{(\mathrm{CH}-\mathrm{AR})}\right)$ magnitudes have a distribution spanning the entire year. To understand the statistical nature of the sample, the distribution is fitted with a gamma function. The probability density function (PDF) for this fit is estimated as

$f(x, s)=\frac{x^{s-1} e^{-x}}{\Gamma(s)}$

for $x \geq 0, s>0$. Here, $s$ is the shape parameter for the gamma function $(\Gamma(s))$.

The mean value of the fitted gamma function was then taken as a threshold to exclude any active regions that were far from the coronal hole center. It should be noted, however, that cases or pairs below the threshold value were taken as a first approximation for our database, although they were also scrutinized by eye, using SDO/AIA imaging data, to filter out any false positives that might have remained in the selected cases. Furthermore, due to the large area of any given coronal hole feature, the estimated distance had magnitudes higher than the threshold values. These pairs, identified in the imaging data, were also included in our list for further analysis.

The list generated had active regions that lie within the close proximity of a coronal hole structure, both inside and outside (at the edge) the coronal hole boundary. Key spatial and magnetic field characteristics for the selected anemone-type features were taken for further analysis. The regions were also checked for any co-spatial filament features and/or flare events using the Heliophysics Events Knowledgebase $\left(\mathrm{HEK}^{3}\right)$ catalog. The nature of the identified flare event, as eruptive or confined was verified using imaging data from SDO/AIA and the online SoHO/LASCO CME $\mathrm{CM}^{4}$ catalog.

Our final database (Sharma \& Cid 2020) had information on location coordinates $(x, y)$, area $(A)$, averaged magnetic field $(|B|)$ for an anemone region, and its host coronal hole, along with the maximum magnetic field magnitude for the active region $\left(B_{\mathrm{AR}(\max )}\right)$. Furthermore, parameters such as the location of an active region with respect to the coronal hole, estimated minimum distance $\left(D_{(\mathrm{CH}-\mathrm{AR})}\right)$, flare magnitude, and presence of

\footnotetext{
https://www . lmsal. com/hek/

4 https://cdaw.gsfc.nasa.gov/CME_list/
}

filament at active region were also taken for our analysis. The ratio(s) of areas $\left(r_{A}\right)$ and average magnetic field $\left(r_{|B|}\right)$ for the anemone regions and the associated coronal holes were also included to get a better understanding of any relation between these physical characteristics. The ratio(s) were given as

$r_{A}=\frac{A_{\mathrm{AR}}}{A_{\mathrm{CH}}}, \quad r_{|B|}=\frac{|B|_{\mathrm{CH}}}{|B|_{\mathrm{AR}}}$.

The area and magnetic field parameters for all identified anemone regions, along with filament and flare information are listed in Table A. $1^{5}$

\section{Results and discussion}

The spatial distribution of all identified anemone regions on the solar disk during the ascending phase of solar cycle 24, in the years 2011-2014, are shown in Fig. 2a. Here the minimum distance $\left(D_{(\mathrm{CH}-\mathrm{AR})}\right)$ between the $\mathrm{COG}$ of an active region and the nearest coronal hole are shown by the relative size of the marker at the location of the anemone region. Most of the anemone regions during the sample interval, irrespective of the $D_{\text {(CH-AR) }}$ parameter, are found located between \pm 500 arcsec from the equator. This is consistent with the fact that the new magnetic field(s) during the rise phase of any solar cycle emerges at higher latitudinal bands and progresses towards the equator with time (Fig. 2a). The set of anemone regions associated with both eruptive and confined flaring events are shown in Fig. 2b, along with the flare magnitude as relative size of the marker. Identified anemone active regions and associated flaring events were found ubiquitously at either side of the solar equator, though the number of such cases in the northern hemisphere $(A R=31$, flares $=11)$ is slightly higher than those in the southern hemisphere $(A R=28$, flares $=7)$, indicating a weak asymmetry in the distribution. A similar bias in the presence of anemone regions in the northern hemisphere is also evident from Asai et al. (2008), where they found around $71 \%$ of their analyzed cases located in the northern hemisphere, despite a greater number of sunspots in the southern hemisphere.

\subsection{Temporal evolution of source-region characteristics}

Physical properties of anemone regions, such as minimum distance $\left(D_{(\mathrm{CH}-\mathrm{AR})}\right)$, area $(A)$, and magnetic field magnitude $(|B|)$, as well as corresponding area $\left(r_{A}\right)$ and magnetic field $\left(r_{|B|}\right)$ ratio(s) were examined to understand the temporal evolution in their characteristics with the rise of the solar cycle. Here, apart from the average magnitudes of magnetic field for the active region and coronal hole features, the maximum magnitude of magnetic field for active region $\left(B_{\mathrm{AR}(\max )}\right)$ is also used in our study. This will help to get a crucial insight into the availability of the maximum field for the reconnection process and subsequent propagation of any ejected field feature along the open magnetic field(s). Figure 3 shows the evolution of anemone-type regions over the years 2011-2014, along with the cases associated with flaring events, indicated by a cross. As a first approximation, there are no apparent trends in the temporal evolution of the anemone region area and magnetic field properties. However, the main characteristics could be better understood by histograms for mean absolute magnitudes for each year, over the rise phase of the solar cycle 24 .

\footnotetext{
5 The detailed catalog is available online with all parameters: https: //doi.org/10.21950/VG1QSK
} 


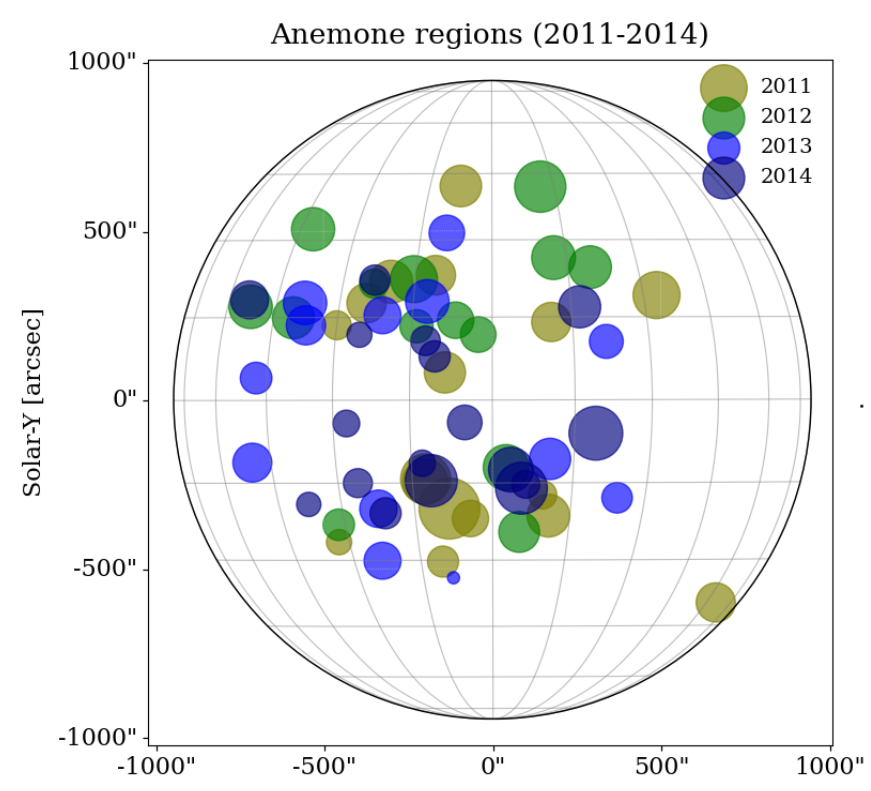

(a) Solar-X [arcsec]

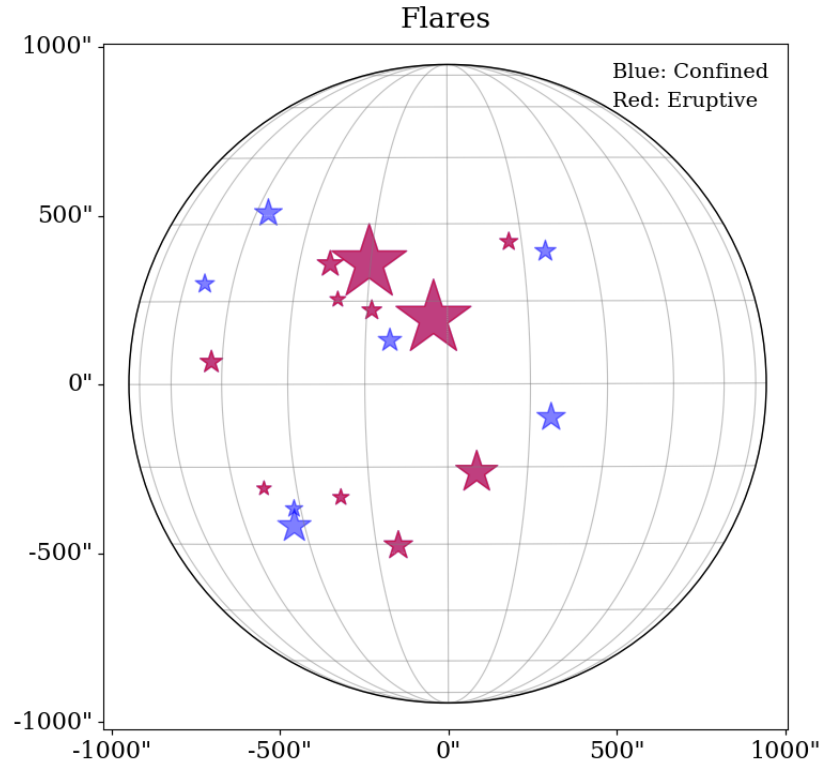

(b) Solar-X [arcsec]

Fig. 2. Heliographic spatial distribution of the anemone active regions and associated flaring events during 2011-2014. Left panel a: location of the anemone regions, with the relative size of the marker in proportion to the estimated distance of the active region from the coronal hole center. Right panel $b$ : anemone regions related with the flare events. The color of the marker indicates the type of the flare as eruptive (red) or confined (blue), while the marker size gives the magnitude of the flare in Geostationary Operational Environmental Satellite (GOES ) soft x-ray (SXR) flux.

Figure 4 provides an overview of the progression during the years 2011-2014 for the number of identified anemone regions and associated filament and flare cases, along with the mean of absolute magnitudes for area and magnetic field parameters for the corresponding years. The mean sunspot number (SSN) shows a clear upward trend with the solar cycle (Fig. 4a), though the number of anemone regions and associated filament and flare events appear to be independent from the SSN behavior. An important facet of this distribution is the association of filaments with the anemone regions. On average, more than one-half $(57.37 \%)$ of the identified anemone regions harbor a filament structure, while only one-third (29.5\%) had any reconnection (flare) event. However, in the year 2012, this percentage was highest (Table 1); over two-thirds of the anemone regions were associated with a filament (78.57\%) and nearly one-half with any flare event $(50.0 \%)$, which included the two most energetic (M class) eruptive flares in our dataset. The link between anemone regions and filaments is also evident from previously reported cases for the anemone regions (Bravo et al. 1998; Chertok et al. 2002; Asai et al. 2009). Among all the reconnection and flare events in our study, a majority of around $88.8 \%$ were $C$ class events (16 out of 18 ), while only a small number of events were associated with M class events ( 2 out of 18). As suggested by Asai et al. (2008), this could possibly be due to the less sheared magnetic configuration of anemone regions, which makes these regions less energetic compared to other active regions elsewhere on the Sun. Interestingly, around $61.11 \%$ (11 out of 18) of these flaring events were eruptive in nature; when coupled to the open magnetic field lines from their associated coronal holes this can strongly influence the near-Earth geomagnetic activity.

Furthermore, the absolute mean of area (Fig. 4b) and estimated minimum-distance parameters (Fig. 4c) show an overall decreasing trend in their magnitudes with the rise in the solar cycle. Apart from an unusual dip in the coronal hole area $\left(A_{\mathrm{CH}}\right)$ in the year 2012, on average the area of anemone active regions and their host coronal hole features continuously shrink in size with the rise in the solar cycle (Table 1). The same is also reflected in the continuous decrease in the mean of estimated area ratio(s) $\left(r_{A}\right)$ parameters. The maximum magnetic field of anemone active regions $\left(|B|_{\mathrm{AR}(\max )}\right)$ shows a gradual increase in its averaged magnitudes (Fig. 4d) with the solar cycle, consistent with the SSN pattern. This trend is also visible in the absolute mean for magnetic field estimates for coronal holes $\left(|B|_{\mathrm{CH}}\right)$, though the same is not evident from the absolute mean for signed magnetic field for the anemone regions $\left(|B|_{\mathrm{AR}}\right)$. An interesting behavior is seen with the area and magnetic field magnitudes for the coronal hole features during 2013-2014. The absolute mean for the magnetic field of coronal hole $\left(|B|_{\mathrm{CH}}\right)$ shows a noticeable increase in estimated magnitude during 2014, which is twice as large as those in the previous year. Conversely, in the same time span, the mean magnitudes for area nearly reduced to half in 2014 compared to 2013.

However, it should be noted that both the coronal hole magnetic field $\left(|B|_{\mathrm{CH}}\right)$ and the signed magnetic field for anemone region $\left(|B|_{\mathrm{AR}}\right)$ parameters suggest a dip in their trends ( $\mathrm{AR}=615.4 \mathrm{G}, \mathrm{CH}=3.4 \mathrm{G}$ ) during 2013, which is in agreement with the study of coronal holes properties during solar cycle 24 by Lowder et al. (2017). These authors highlighted the onset of polarity reversals for coronal holes in 2012 for the northern hemisphere and 2013 for the southern hemisphere. Moreover, they also identified a drop in magnetic fields for coronal holes in the southern hemisphere, which could possibly explain the overall reduction in magnetic field associated with the anemone regions in 2013.

\subsection{Distribution of physical characteristics}

The distribution of spatial and magnetic field magnitudes, with estimated mode values for flaring and non-flaring anemone 


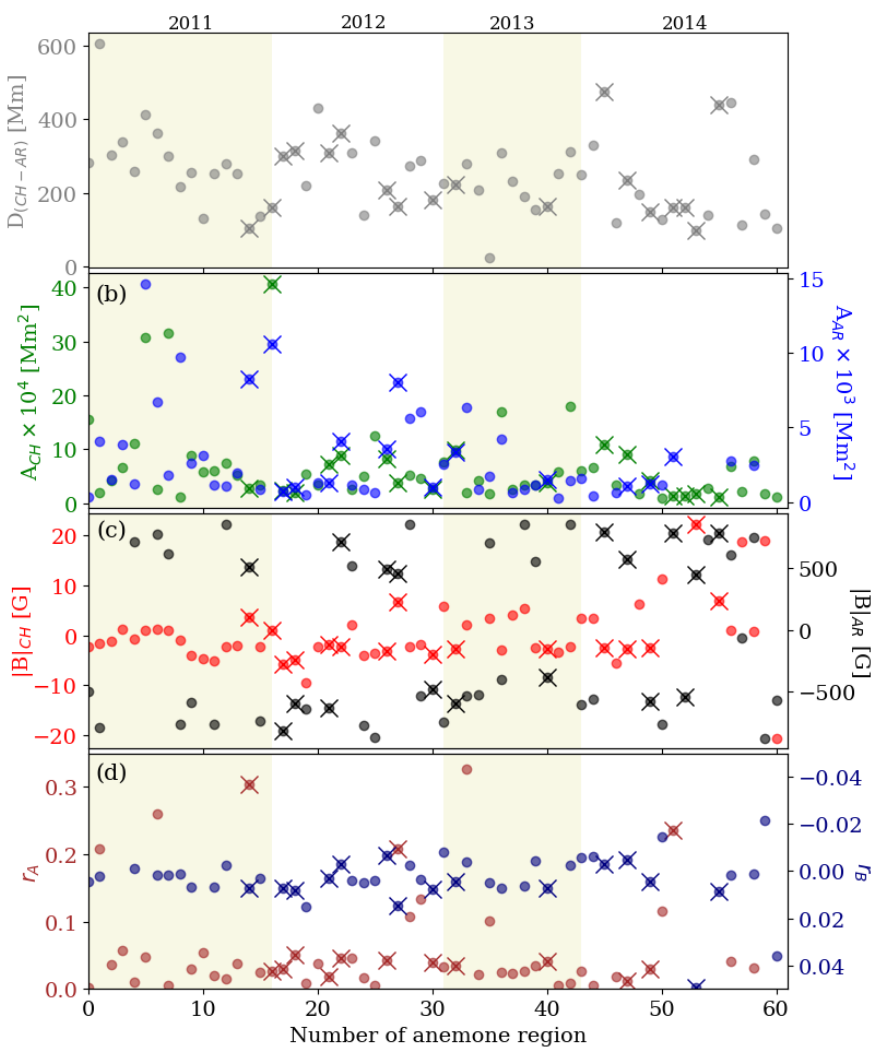

Fig. 3. Temporal evolution of the physical parameters of anemone active regions and associated coronal hole features during the years 20112014. From top to bottom: $(a)$ minimum distance $\left(D_{(\mathrm{CH}-\mathrm{AR})}\right)$ between the center of gravity of anemone active regions and their host coronal holes, $(b)$ total area magnitudes $(A),(c)$ magnetic field strength $(|B|)$, and estimated ratios for area $\left(r_{A}\right)$ and magnetic field $\left(r_{B}\right)$ parameters $(d)$. Magnitudes of estimated parameters associated with flaring events are overplotted with a cross $(\times)$.

regions and their host coronal hole features are given in Fig. 5. A comparison of properties of flaring and non-flaring regions (Table 2) can further help to understand the relationship between an anemone region and its surrounding, and also in determining its ability to produce an energetic event. An overview of the distribution suggests notable differences in mode values for flaring and non-flaring regions in both area and magnetic field parameters. The estimated minimum distance $\left(D_{(\mathrm{CH}-\mathrm{AR})}\right)$ indicates a rather symmetric distribution of magnitudes (Fig. 5a) with the mode of the total sample located at $279.4 \mathrm{Mm}$. The flaring anemone regions, in general, were located much closer to the host coronal hole centers with mode value of $167.9 \mathrm{Mm}$ compared to the non-flaring regions, which had a similar mode value of $279.4 \mathrm{Mm}$, as an overall sample.

The histograms for active region and coronal hole area magnitudes and their respective ratio(s) (Figs. 5b-c) suggest a positive (right-handed) skewness in its distributions. The estimated mode values for area parameters indicate slightly higher magnitudes for anemone regions associated with a reconnection event compared to the non-flaring regions. Coronal hole area $\left(A_{\mathrm{CH}}\right)$ for flaring anemone regions (mode $\left.=3.7 \times 10^{4} \mathrm{Mm}^{2}\right)$ are larger $\left(\sim 0.8 \times 10^{4} \mathrm{Mm}^{2}\right)$ than those without a flare $\left(\right.$ mode $\left.=2.9 \times 10^{4} \mathrm{Mm}^{2}\right)$, with an overall mode value of $3.5 \times 10^{4} \mathrm{Mm}^{2}$ for the total sample used in our study. A similar aspect is also found for the active region areas $\left(\right.$ mode $\left.=1200.41 \mathrm{Mm}^{2}\right)$ where the flaring anemone regions $\left(\right.$ mode $\left.=1351.1 \mathrm{Mm}^{2}\right)$ had a larger size $\left(\sim 150 \mathrm{Mm}^{2}\right)$ compared to the non-flaring anemone regions (mode $=1200.4 \mathrm{Mm}^{2}$ ). However, the ratio of active region to coronal hole areas $\left(r_{A}\right)$ indicates a significant difference in area for flaring anemone regions from those without flare events, as reflected by the lower mode value for flaring anemone regions (0.03) than for non-flaring regions (0.054).

In contrast to area magnitudes, both the signed and unsigned magnetic field of anemone regions and coronal holes appear to be symmetrically distributed (Figs. $5 \mathrm{e}-\mathrm{g}$ ). The magnetic field distribution for coronal hole features $\left(|B|_{\mathrm{CH}}\right)$ suggests that most of the anemone features are associated with negative polarity regions (Table 2), for both flaring and non-flaring cases. This is contrary to the study from Asai et al. (2008), where most of the anemone active regions emerge within the positive polarity coronal hole features. The estimated mode value for coronal holes associated with flaring anemone regions was higher $(-4.04 \mathrm{G})$ than those for non-flaring regions $(-3.21 \mathrm{G})$, with a mode value of total sample around $-1.77 \mathrm{G}$.

For active regions, the signed magnetic field appears to be symmetrically distributed at both polarities (Fig. 5f), though the number of regions associated with negative polarity field were dominant, with an exception for those regions that were related with flaring events. In either magnetic polarity active region, the mode value for flaring anemone sites was lower compared to non-flaring regions. In positive polarity active regions, the mode for flaring anemone region has the value of $717.0 \mathrm{G}$, while nonflaring regions had the value of $775.02 \mathrm{G}$. Similarly, for negative polarity anemone regions, flaring sites had the mode value of $576.15 \mathrm{G}$ compared to non-flaring regions with the mode value of $-782.02 \mathrm{G}$.

The histogram for maximum magnitudes of anemone region magnetic field $\left(|B|_{\mathrm{AR}(\max )}\right)$ indicate a multi-modal distribution of values. Contrary to signed magnetic fields, the peak mode values for $|B|_{\mathrm{AR}(\max )}$ show an interesting tendency of higher mode values $(1461.5 \mathrm{G})$ for flaring regions, compared to non-flaring regions $(1379.9 \mathrm{G})$, with an overall mode value of $1236.7 \mathrm{G}$ for the sample. This gives an indication that the maximum available magnetic field for an active region together with the field of the corresponding host coronal hole are crucial factors in determining the flaring capability of the anemone regions. The ratio of averaged magnetic fields (Fig. 5h), however, had a marginally lower magnitude for flaring regions (0.0074) than for non-flaring regions $(-0.014)$. It should be noted that all the anemone regions associated with reconnection events, both eruptive and confined, had positive magnitudes for the magnetic field ratios compared to non-flaring regions.

\subsection{Mutual-relationship between estimated parameters}

Figure 6 displays the 2D density estimates for spatial and magnetic field characteristics used in our study, which help to better understand the mutual relationship between these parameters at flaring and non-flaring anemone sites. The concentrations of peak densities of probability distributions estimates for corresponding pairs of minimum distance, area, and magnetic field for active regions (ARs) and associated coronal holes (CHs) highlight clear discrepancies for anemone regions with (FL) and without (NF) reconnection events. Density concentrations for minimum-distance $\left(D_{(\mathrm{CH}-\mathrm{AR})}\right)$ and area $\left(A_{\mathrm{AR}}, A_{\mathrm{CH}}\right)$ parameters (Figs. 6a-d) for both active regions and host coronal holes suggest anemone regions with reconnection or flare events had 

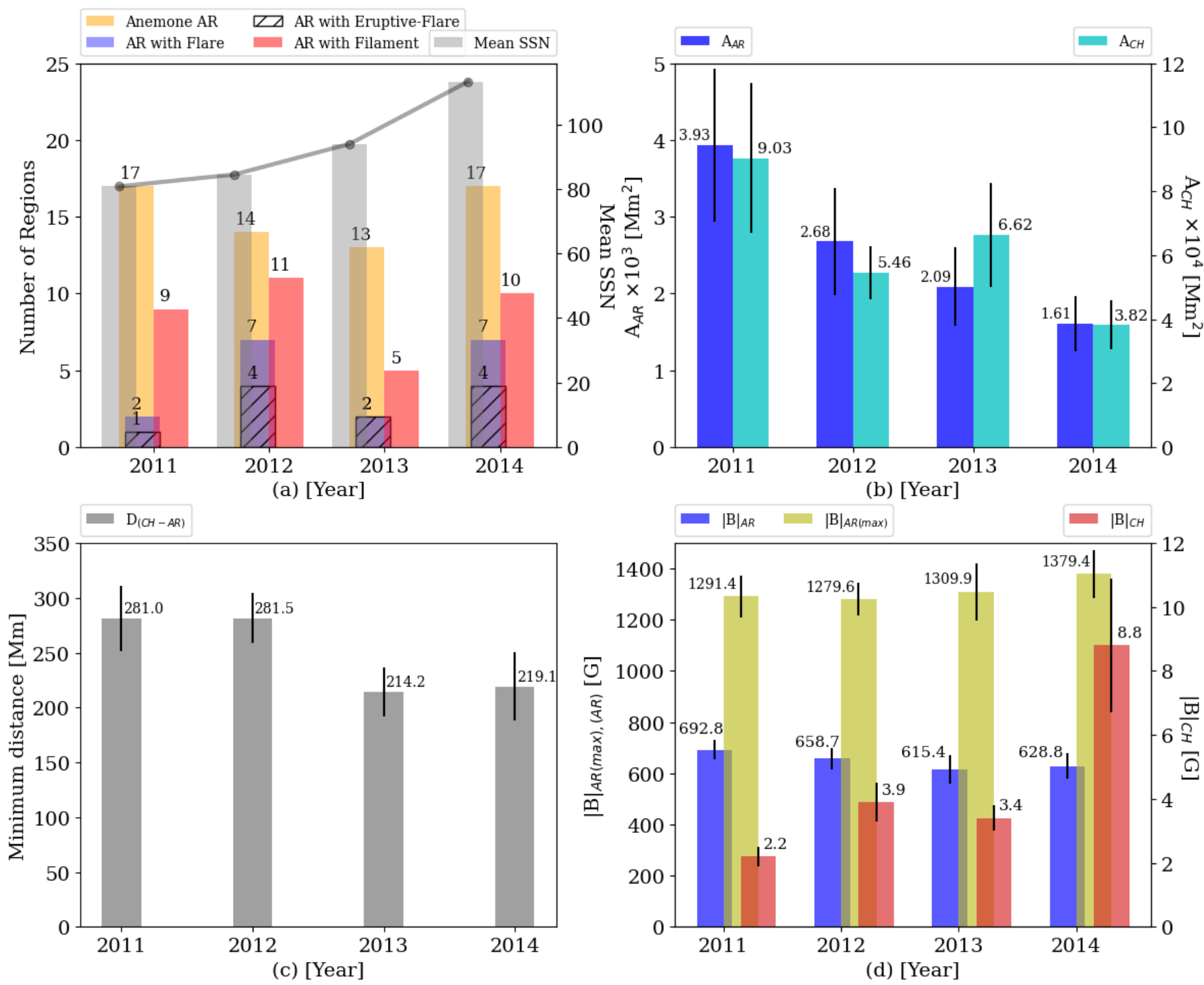

Fig. 4. Distributions of average magnitudes of spatial and magnetic field properties for anemone regions during the ascending phase of solar cycle 24. From left to right: (a) average sunspot numbers for the years 2011-2014 indicating a steady rise in solar activity with counts of identified anemone regions, along with those associated with flare (eruptive as shaded region) and filament features; $(b)$ averaged magnitudes of area parameters for anemone active regions and their host coronal hole, with standard error in estimation (shown as vertical bars) for each year; (c) estimated minimum-distance parameter with error bars during 2011-2014. Panel $d$ : absolute mean of magnetic field magnitudes for anemone regions and nearest coronal holes, along with mean for the maximum magnitude of the magnetic field for the active regions.

larger area $\left(\mathrm{AR}=[\mathrm{FL}: 2.1, \mathrm{NF}: 1.3] \times 10^{3} \mathrm{Mm}^{2}, \mathrm{CH}=[\mathrm{FL}: 6.0\right.$, $\left.\mathrm{NF}: 5.22] \times 10^{4} \mathrm{Mm}^{2}\right)$ and smaller distance $(\mathrm{AR}=[\mathrm{FL}: 232.4$, $\mathrm{NF}: 245.5] \mathrm{Mm}, \mathrm{CH}=[\mathrm{FL}: 194.1, \mathrm{NF}: 245.5] \mathrm{Mm})$ between the COG of corresponding features.

However, in case of minimum-distance and magnetic field characteristics, both $\mathrm{AR}$ and $\mathrm{CH}$ features indicate the location of flaring anemone regions closer to the host (Figs. 6e-h) compared to non-flaring regions. On average, flaring active regions and coronal holes were located at a distance of $181.3 \mathrm{Mm}$ from each other, while non-flaring regions were present at $265.6 \mathrm{Mm}$ (AR), and $285.7 \mathrm{Mm}(\mathrm{CH})$. Interestingly, the maximum magnitude of active region magnetic field $\left(|B|_{\mathrm{AR}(\max )}\right)$ had similar magnitudes $\left(13.3 \times 10^{2} \mathrm{G}\right)$ for both flaring and non-flaring regions, though $\mathrm{CH}$ magnetic field estimates indicate a higher magnitude for flaring $(3.2 \mathrm{G})$ compared to non-flaring $(1.9 \mathrm{G})$ cases.

A comparison of area and magnetic field densities suggest that flaring regions clearly had larger areas (Figs. 6i-1) for all AR and $\mathrm{CH}$ features than non-flaring regions. In general, anemone regions and their host coronal holes with reconnection events had areas $\sim 1.6$ times larger $\left(\mathrm{AR}=[\mathrm{FL}: 2.1 \mathrm{NF}: 1.3] \times 10^{3} \mathrm{Mm}^{2}, \mathrm{CH}=\right.$ [FL:7.3, NF:4.3] $\times 10^{4} \mathrm{Mm}^{2}$ ) than those without any flare events. This distinction is also evident from the magnetic field densities, where flaring anemone regions had slightly higher (AR = [FL:13.2, NF:13.1] $\times 10^{2} \mathrm{G}$ ) magnitudes, but in the case of coronal hole magnetic fields the features associated with reconnection events had higher ( $\mathrm{CH}=[\mathrm{FL}: 2.8, \mathrm{NF}: 2.2] \mathrm{G})$ magnitudes of available field, indicating a stronger role of open-field line topology in flaring events related to anemone regions. The results for mutual relationship analysis are summarized in Table 3.

\section{Conclusions}

The main goal of this work was to examine the key spatial and magnetic field characteristics of anemone active regions and their associated coronal hole structures. Available online catalogs from multiple sources were used for our study. Around 
R. Sharma and C. Cid: Source-region characteristics of anemone active regions in the ascending phase of solar cycle 24

Table 1. Summary of mean of absolute values for magnetic field and spatial parameters of anemone regions and host coronal holes, along with the percentage of regions with associated filament and flare features during the years 2011-2014.

\begin{tabular}{|c|c|c|c|c|c|c|c|c|c|c|}
\hline \multirow[t]{2}{*}{ Year } & \multicolumn{3}{|c|}{ Magnetic field [G] } & \multicolumn{3}{|c|}{ Spatial (Minimum distance/Area) $)^{(*)}$} & \multicolumn{2}{|c|}{ Ratio } & \multirow[t]{2}{*}{$\%$ Fil. } & \multirow[t]{2}{*}{$\%$ Flare } \\
\hline & $|B|_{\mathrm{CH}}$ & $|B|_{\mathrm{AR}}$ & $|B|_{\mathrm{AR}(\max )}$ & $D_{(\mathrm{CH}-\mathrm{AR})}$ & $A_{\mathrm{CH}}\left(\times 10^{4}\right)$ & $A_{\mathrm{AR}}\left(\times 10^{3}\right)$ & $r_{A}$ & $r_{|B|}$ & & \\
\hline & $2.2 \pm 0.3$ & $692.8 \pm 36.2$ & $291.4 \pm 82.7$ & $81.0 \pm 30.0$ & $9.03 \pm 2.33$ & $3.93 \pm 0.99$ & .122 & 0.004 & 52.94 & 11.76 \\
\hline & & .8 & 1279 & 22.6 & & .69 & 0.058 & 0.006 & 78.57 & 50.0 \\
\hline & $3.4 \pm($ & 615.4 & 1309. & & & & 0.057 & 0.005 & 38. & 15.38 \\
\hline & & & & & & & 0.061 & 0.038 & 58.82 & 41.17 \\
\hline Average & $4.5 \pm 0.6$ & $643.8 \pm 22.8$ & $1314.1 \pm 43.1$ & $246.5 \pm 13.9$ & $6.71 \pm 0.95$ & $2.86 \pm 0.41$ & 0.016 & 0.014 & 57.37 & 29.50 \\
\hline
\end{tabular}

Notes. ${ }^{(*)}$ The minimum distance $\left(D_{(\mathrm{CH}-\mathrm{AR})}\right)$ magnitudes is in units of $\mathrm{Mm}$, while the average area parameters for coronal holes $\left(A_{\mathrm{CH}}\right)$ and anemone active regions $\left(A_{\mathrm{AR}}\right)$ are in units of $\mathrm{Mm}^{2}$. The standard error of the measurement is taken as $\pm \sigma$ for all estimated parameters.
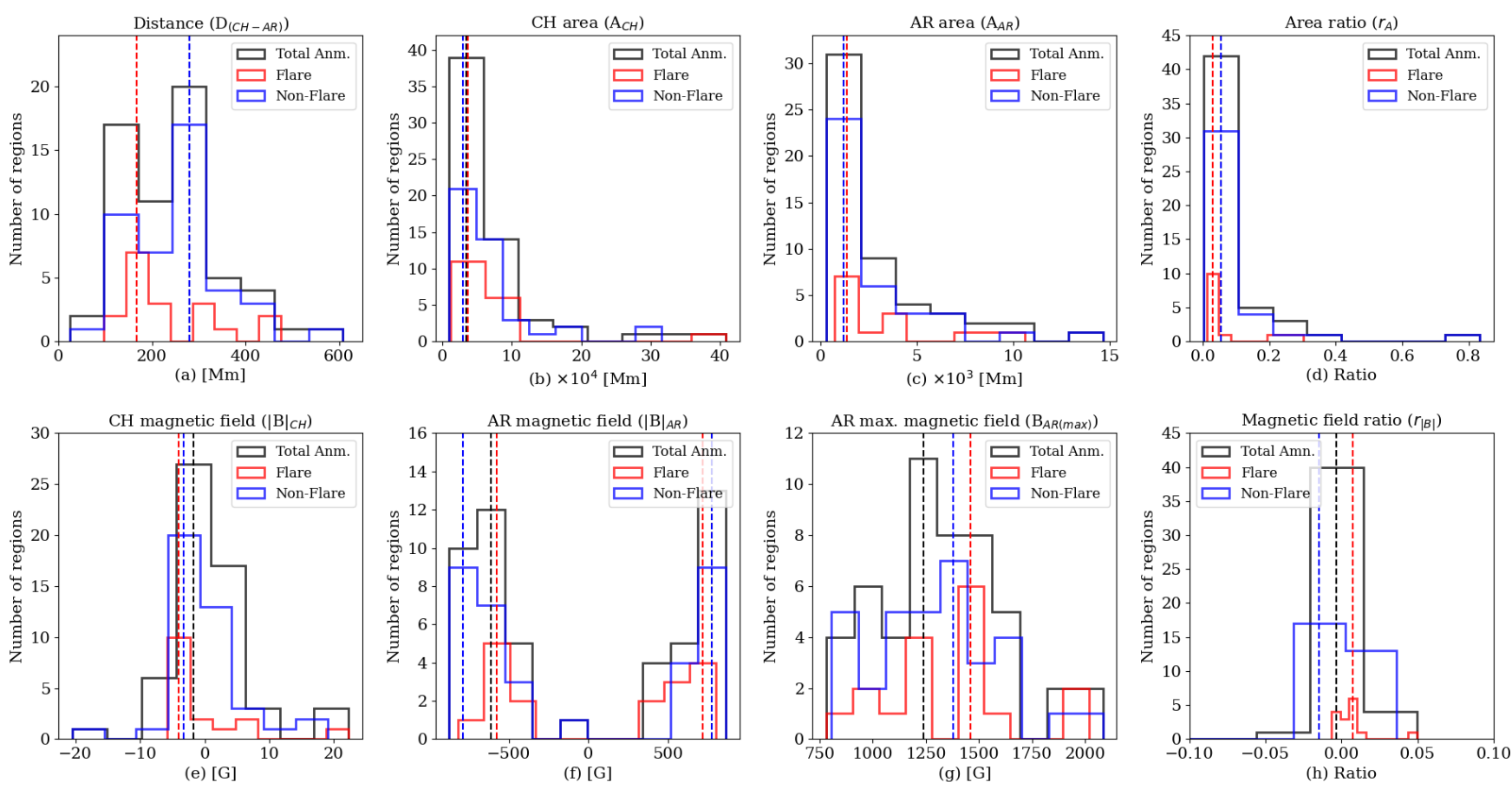

Fig. 5. Distribution of spatial (panels $a-d$ ) and magnetic field (panels $e-h$ ) characteristics for identified anemone regions (black), along with the cases associated with (red) and without (blue) a reconnection or flare event. Shown are the minimum distance $D_{(\mathrm{CH}-\mathrm{AR})}\left(\mathrm{panel} a\right.$ ), area of $A_{\mathrm{CH}}$ and $A_{\mathrm{AR}}$ (panels $b$ and $c$, respectively), area ratio $\left(r_{A}\right)$ (panel $d$ ), and averaged magnetic fields $|B|_{\mathrm{CH}}$ and $|B|_{\mathrm{AR}}$ (panels e and $f$, respectively). Panel $g$ displays the maximum magnitude of available magnetic field at the active region $B_{\mathrm{AR}(\max )}$, while the magnetic field ratio $r_{|B|}$ for averaged magnitudes of active region and coronal hole magnetic fields are shown in panel $h$. Mode values for all the estimated parameters for total, flaring, and non-flaring cases are highlighted by dashed lines.

Table 2. Distribution counts and associated mode values of spatial and magnetic field characteristics for non-flaring and flaring anemone regions.

\begin{tabular}{|c|c|c|c|c|c|c|}
\hline \multirow[t]{2}{*}{ Parameter(s) } & \multicolumn{2}{|c|}{ All anemone regions } & \multicolumn{2}{|c|}{ Non-flaring regions } & \multicolumn{2}{|r|}{ Flaring regions } \\
\hline & Counts & Mode & Counts & Mode & Counts & Mode \\
\hline$D_{\mathrm{CH}-\mathrm{AR}}$ & 61 & 279.4 & 43 & 279.4 & 18 & 167.9 \\
\hline$A_{\mathrm{CH}}$ & 61 & 3.5 & 43 & 2.9 & 18 & 3.7 \\
\hline$A_{\mathrm{AR}}$ & 52 & 1.2 & 38 & 1.2 & 14 & 1.35 \\
\hline$r_{A}$ & 52 & 0.054 & 38 & 0.054 & 14 & 0.03 \\
\hline$|B|_{\mathrm{CH}}$ & 57 & 1.77 & 41 & 3.21 & 16 & 4.04 \\
\hline$|B|_{\mathrm{AR}}$ & $(P: 22, N: 28)$ & $(P=775.02, N=-613.46)$ & $(P: 13, N: 20)$ & $(P=775.02, N=-782.02)$ & $(P: 9, N: 8)$ & $(P=717.0, N=-576.15)$ \\
\hline$|B|_{\mathrm{AR}(\max )}$ & 50 & 1236.7 & 33 & 1379.9 & 17 & 1461.5 \\
\hline$\underline{r_{|B|}}$ & 46 & -0.0034 & 31 & -0.0014 & 15 & 0.0074 \\
\hline
\end{tabular}

Notes. The minimum-distance paramater $\left(D_{(\mathrm{CH}-\mathrm{AR})}\right)$ is in units of $\mathrm{Mm}$, while the mode values of area parameters in units for coronal holes $\left(A_{\mathrm{CH}} \times 10^{4} \mathrm{Mm}^{2}\right)$ and anemone active regions $\left(A_{\mathrm{AR}} \times 10^{3} \mathrm{Mm}^{2}\right)$, respectively. Magnetic field parameters $\left(|B|_{\mathrm{CH}},|B|_{\mathrm{AR}},|B|_{\mathrm{AR}(\max )}\right)$ are expressed in units of G. Also, " $P$ " and " $N$ " respectively denote the positive $(+)$ and negative $(-)$ polarities associated with the selected regions. 

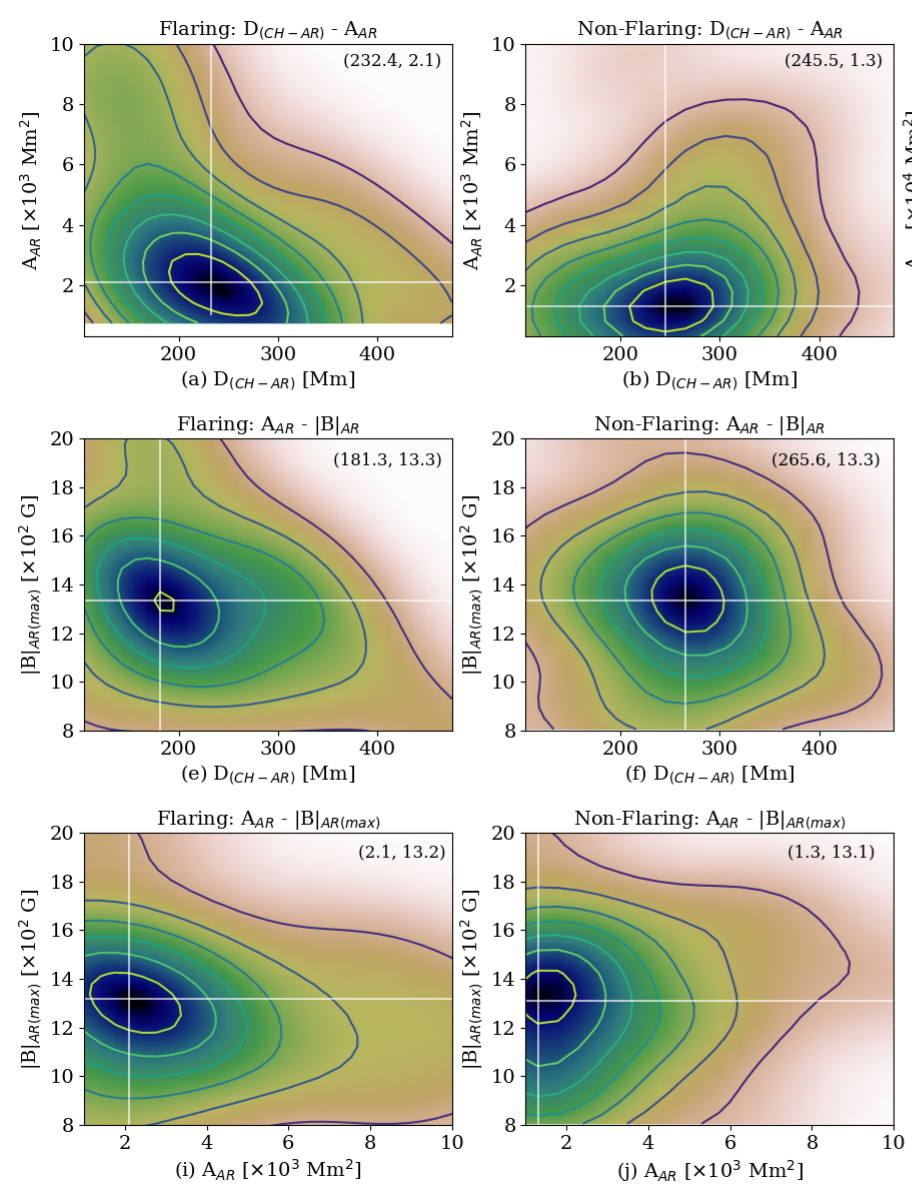
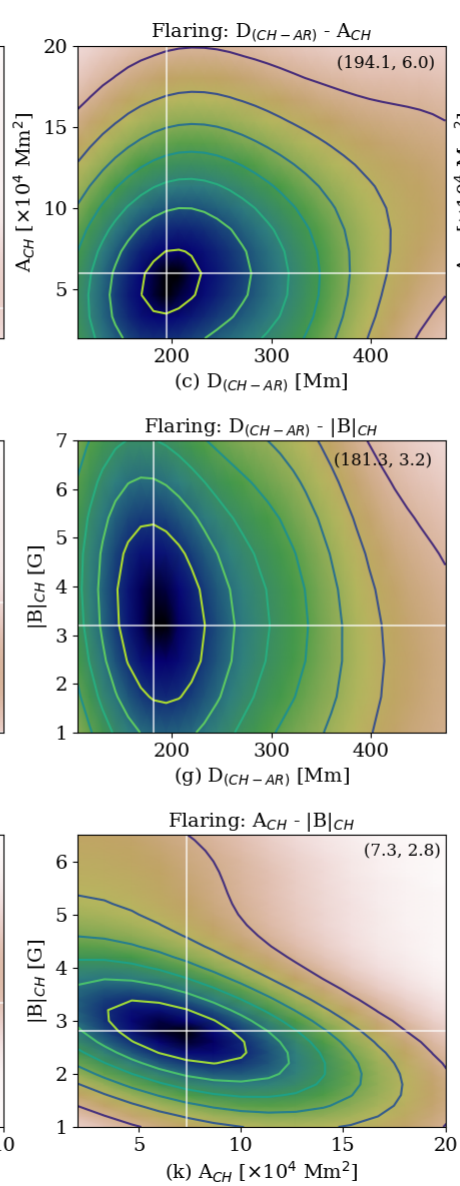
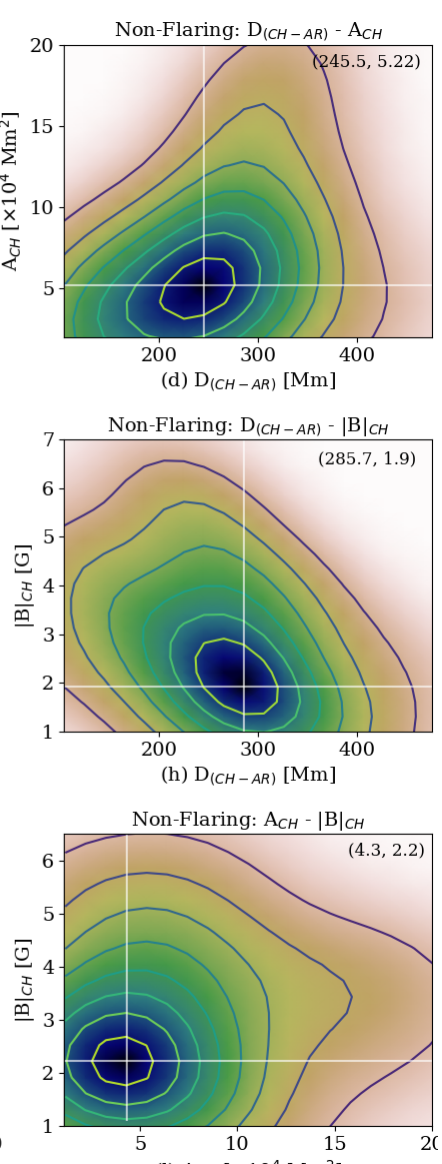

Fig. 6. Two-dimensional density plots indicate the mutual relationship between estimated spatial and magnetic field parameters for flaring and non-flaring anemone active regions and coronal holes. The top panels $a-d$ show probability densities of area and minimum-distance parameters, while the middle panels $e-h$ highlight the mutual variations in magnetic field and minimum-distance estimates. The bottom panels $i-l$ highlight the relationship between magnetic field and area parameters of anemone AR and $\mathrm{CH}$, with and without flares. Peak magnitudes for corresponding parameters are annotated in each plot.

Table 3. Summary of estimated probability density range highlighting mutual relationships between the distance, area and magnetic field parameters for flaring and non-flaring anemone regions as shown in Fig. 6.

\begin{tabular}{lccc}
\hline \hline \multirow{2}{*}{ Features } & \multicolumn{3}{c}{ Parameters } \\
\cline { 2 - 4 } & $D_{(\mathrm{CH}-\mathrm{AR})}$ & $A$ & $|B|$ \\
\hline Flaring AR & $181.3-232.4$ & 2.1 & $13.2-13.3$ \\
Non-flaring AR & $245.6-265.6$ & 1.3 & $13.1-13.3$ \\
Flaring CH & $181.3-194.1$ & $6.0-7.3$ & $2.8-3.2$ \\
Non-flaring CH & $245.5-285.7$ & $4.3-5.22$ & $1.9-2.22$ \\
\hline
\end{tabular}

Notes. Here, minimum distance $\left(D_{(\mathrm{CH}-\mathrm{AR})}\right)$, area $(A)$ and magnetic field $(|B|)$ parameters have units expressed in "Mm", "Mm" " and "G" respectively. Area estimates for AR and $\mathrm{CH}$ has order of magnitude as $\times 10^{3}$ and $\times 10^{4}$, while AR magnetic field has magnitudes of the order $\times 10^{2}$.

61 anemone-type active regions were identified, based on the estimated minimum distance from the center of their host coronal holes, during the ascending phase of solar cycle 24 . Averaged area and magnetic field (signed and unsigned) parameters, along with their ratios were further investigated to better understand the properties of flaring and non-flaring anemone regions. The major findings from our study are as follows:
1. Weak asymmetry in the spatial distribution of anemone regions is apparent with a higher number of cases (active regions and flares) in the northern hemisphere. On average, more than half $(57.3 \%)$ of the identified anemone cases had a filament structure, while only a third (29.5\%) were associated with a magnetic reconnection (flare) event. However, a peculiarity is evident for the year 2012: the percentage of regions associated with any filament $(78.57 \%)$ and/or flare events $(50.0 \%)$ was highest.

2. Around $61.11 \%$ of the identified flare events were eruptive in nature, as suggested from SDO/AIA imaging and $\mathrm{SoHO} / \mathrm{LASCO}$ data. This could possibly be due to the interchange reconnection mechanism between closed (active region) and open (coronal hole) magnetic field topologies, facilitating the escape of magnetic flux in interplanetary environment, with strong implications for near-Earth geomagnetic activities.

3. Yearly averaged magnitudes of spatial parameters (minimum distance and area), for anemone active regions and coronal holes show a decreasing trend with the rise in the solar cycle. However, an opposite (increasing) trend is evident from the mean of magnetic field characteristics (coronal hole, maximum magnitude of field for active regions), which is consistent with the rise in sunspot numbers during 2011-2014. 
4. A clear distinction in area and magnetic field properties for flaring and non-flaring anemone regions is noticeable from the estimated mode values for the distribution. Anemone regions associated with any flare event were closer to the center of the host coronal hole than the non-flaring regions. Also, the magnitude of area of flaring active regions and their coronal hole was higher compared to the non-flaring active regions. Most of the anemone regions and their companion coronal holes were predominantly negative polarity regions; the flaring active regions had higher magnitudes of coronal hole $\left(|B|_{\mathrm{CH}}\right)$ and active region magnetic field $\left(|B|_{\mathrm{AR}(\max )}\right)$.

5. Probability density estimates of area, distance, and magnetic field pairs support the above conclusion with most of the flaring anemone regions having larger area and magnetic field, and a smaller distance between the anemone active region and its host coronal hole feature.

Our study provides a vital overview of the source-region characteristics of anemone regions for a limited time interval during the ascending phase of solar cycle 24 . In the future, a more comprehensive study with a comparison of anemone region properties for multiple solar cycles will provide a more detailed insight into the variations of their on-disk properties, along with possible effects on interplanetary solar wind conditions. Investigation of specific anemone regions for the aforementioned objectives are planned and will be published in subsequent articles.

Acknowledgements. The authors would like to thank the respective teams of Solar and Space Weather Network of Excellence (SOLSPANET), Heliophysics Feature Catalogue (HFC) and Heliophysics Events Knowledgebase (HEK) for compiling and providing the catalogs online. R. S. and C. C. acknowledges support by the Spanish Ministry of Economy and Competitiveness (MINECO) through project AYA2016-80881-P (including FEDER funds). This research has made use of NASA's Astrophysics Data System Bibliographic Services. We acknowledge the community effort devoted to the development of the following open-source packages that were used in this work: Numpy (numpy . org), Pandas (pandas.pydata.org), Matplotlib (matplotlib.org), pfsspy (Stansby et al. 2019), Sunpy (The SunPy Community 2020) and Scipy (scipy.org).

\section{References}

Asai, A., Shibata, K., Hara, H., \& Nitta, N. V. 2008, ApJ, 673, 1188
Asai, A., Shibata, K., Ishii, T. T., et al. 2009, J. Geophys. Res. (Space Phys.), 114, A00A21

Bagashvili, S. R., Shergelashvili, B. M., Japaridze, D. R., et al. 2017, A\&A, 603, A 134

Barra, V., Delouille, V., Kretzschmar, M., \& Hochedez, J. F. 2009, A\&A, 505, 361

Bravo, S., Cruz-Abeyro, J. A. L., \& Rojas, D. 1998, Ann. Geophys., 16, 49

Chertok, I. M., Obridko, E. I., Mogilevsky, V. N., Shilova, N. S., \& Hudson, H. S. 2002, ApJ, 567, 1225

Cranmer, S. R. 2009, Liv. Rev. Sol. Phys., 6, 3

Crooker, N. U., \& Owens, M. J. 2012, Space Sci. Rev., 172, 201

Gopalswamy, N. 2006, Space Sci. Rev., 124, 145

Gopalswamy, N., Mäkelä, P., Xie, H., Akiyama, S., \& Yashiro, S. 2009, J. Geophys. Res. (Space Phys.), 114, A00A22

Grandin, M., Aikio, A. T., \& Kozlovsky, A. 2019, J. Geophys. Res. (Space Phys.), 124, 3871

He, J. S., Marsch, E., Tu, C. Y., Guo, L. J., \& Tian, H. 2010, A\&A, 516, A14

Heinemann, S. G., Temmer, M., Farrugia, C. J., et al. 2019a, Sol. Phys., 294, 121

Heinemann, S. G., Temmer, M., Heinemann, N., et al. 2019b, Sol. Phys., 294, 144

Janardhan, P., Tripathi, D., \& Mason, H. E. 2008, A\&A, 488, L1

Kilpua, E. K. J., Balogh, A., von Steiger, R., \& Liu, Y. D. 2017, Space Sci. Rev., 212, 1271

Kumar, P., Karpen, J. T., Antiochos, S. K., et al. 2019, ApJ, 873, 93

Lemen, J. R., Title, A. M., Akin, D. J., et al. 2012, Sol. Phys., 275, 17

Lowder, C., Qiu, J., \& Leamon, R. 2017, Sol. Phys., 292, 18

Macneil, A. R., Owen, C. J., Baker, D., et al. 2019, ApJ, 887, 146

Mäkelä, P., Gopalswamy, N., Xie, H., et al. 2013, Sol. Phys., 284, 59

Moreno-Insertis, F., \& Galsgaard, K. 2013, ApJ, 771, 20

Nishizuka, N., Nakamura, T., Kawate, T., Singh, K. A. P., \& Shibata, K. 2011, ApJ, 731, 43

Pesnell, W. D., Thompson, B. J., \& Chamberlin, P. C. 2012, Sol. Phys., 275, 3

Priest, E., \& Forbes, T. 2000, Magnetic Reconnection (UK: Cambridge University Press)

Schrijver, C. J., \& De Rosa, M. L. 2003, Sol. Phys., 212, 165

Sharma, R., \& Cid, C. 2020, Anemone Active Regions Catalogue from 2011 2014 (e-cienciaDatos)

Shibata, K., Nitta, N., Strong, K. T., et al. 1994, ApJ, 431, L51

Shibata, K., Nakamura, T., Matsumoto, T., et al. 2007, Science, 318, 1591

Shugay, Y., Slemzin, V., Rodkin, D., Yermolaev, Y., \& Veselovsky, I. 2018, J. Space Weather Space Clim., 8, A28

Singh, K. A. P., Shibata, K., Nishizuka, N., \& Isobe, H. 2011, Phys. Plasmas, 18, 111210

Stansby, D., Horbury, T. S., Wallace, S., \& Arge, C. N. 2019, Res. Notes Am. Astron. Soc., 3, 57

The SunPy Community 2020, ApJ, 890, 68

Toriumi, S., \& Wang, H. 2019, Liv. Rev. Sol. Phys., 16, 3

Tousey, R., Bartoe, J. D. F., Bohlin, J. D., et al. 1973, Sol. Phys., 33, 265 
Appendix A: Brief list of identified anemone-type active regions during ascending phase of solar cycle 24, in the years 2011-2014

Table A.1. Source-region characteristics of identified anemone regions during 2011-2014.

\begin{tabular}{|c|c|c|c|c|c|c|c|c|c|c|c|c|c|}
\hline \multirow[t]{2}{*}{ Date } & \multirow{2}{*}{$\begin{array}{c}\mathrm{CH} \\
\text { (SPoCA) }\end{array}$} & \multicolumn{2}{|c|}{$\mathrm{CH}$ loc. } & \multirow{2}{*}{$\begin{array}{c}A_{\mathrm{CH}} \\
\left(\mathrm{Mm}^{2}\right) \\
\end{array}$} & \multirow{2}{*}{$\begin{array}{c}|B|_{\mathrm{CH}} \\
(\mathrm{G}) \\
\end{array}$} & \multirow{2}{*}{$\begin{array}{c}\text { AR } \\
\text { (NOAA) }\end{array}$} & \multicolumn{2}{|c|}{ AR loc. } & \multirow{2}{*}{$\begin{array}{c}A_{\mathrm{AR}} \\
\left(\mathrm{Mm}^{2}\right) \\
\end{array}$} & \multirow{2}{*}{$\begin{array}{c}|B|_{\mathrm{AR}} \\
(\mathrm{G}) \\
\end{array}$} & \multirow{2}{*}{$\begin{array}{c}|B|_{\mathrm{AR}(\max )} \\
(\mathrm{G})\end{array}$} & \multirow[t]{2}{*}{ Flare } & Fil \\
\hline & & $x_{\mathrm{CH}}$ & $y_{\mathrm{CH}}$ & & & & $x_{\mathrm{AR}}$ & $y_{\mathrm{AR}}$ & & & & & \\
\hline 2011-01-05 & 1249 & 128.42 & 310.12 & 155471 & -2.3 & 11140 & -91.9 & 633.12 & 340.13 & 1869.11 & -497.69 & - & $\mathrm{Y}$ \\
\hline 2011-01-08 & 1261 & -687.9 & 301.1 & 19538.5 & -1.7 & 11143 & -128.02 & -322.16 & 4060.69 & 1179.9 & -790.86 & - & $\mathrm{N}$ \\
\hline 2011-01-11 & 1261 & 108.4 & 252.24 & 42375.8 & -1.1 & 11145 & -299.18 & 350.04 & 1524.93 & - & - & - & $\mathrm{N}$ \\
\hline 2011-02-10 & 1434 & -125.69 & -702.36 & 66620.3 & 1.3 & 11156 & -199.9 & -242.18 & 3866.88 & - & - & - & $\mathrm{N}$ \\
\hline 2011-03-01 & 1509 & 176.29 & 460.65 & 110514 & -0.7 & 11163 & -166.88 & 369.14 & 1198.76 & 1062.86 & 712 & - & $\mathrm{Y}$ \\
\hline 2011-03-12 & 1515 & 66.11 & -738.01 & 306784 & 1.0 & 11171 & -198.44 & -234.18 & 14641.3 & - & - & - & $\mathrm{Y}$ \\
\hline 2011-03-19 & 1608 & 16.89 & 480.72 & 25798.2 & 1.3 & 11175 & 487.94 & 308.7 & 6705.97 & 1229.9 & 775.4 & - & $\mathrm{N}$ \\
\hline 2011-04-09 & 1739 & 12.56 & -728.14 & 315085 & 1.1 & 11188 & 165.61 & -343.66 & 1839.7 & 1187.24 & 621.03 & - & $\mathrm{Y}$ \\
\hline 2011-04-26 & 1802 & -89.46 & -54.14 & 11638.6 & -1.0 & 11196 & -65.72 & -351.78 & 9701.02 & 1129.88 & -766.95 & - & $\mathrm{Y}$ \\
\hline 2011-05-30 & 1964 & -190.14 & 587.61 & 88207.3 & -4.0 & 11225 & -372.45 & 287.06 & 2606.21 & 1644.23 & -586.68 & - & $\mathrm{Y}$ \\
\hline 2011-06-21 & 2074 & 327.93 & -252.94 & 58089.5 & -4.6 & 11238 & 148.5 & -281.85 & 3123.99 & - & - & - & $\mathrm{N}$ \\
\hline 2011-06-29 & 2099 & 87.02 & 567.42 & 59336.9 & -5.1 & 11242 & 175.47 & 229.83 & 1139.25 & 1439.08 & -763.09 & - & Y \\
\hline 2011-07-17 & 2203 & 64.13 & -244.38 & 74022.1 & -2.2 & 11256 & -140.52 & 81.11 & 1106.38 & 1415.43 & 861.8 & - & Y \\
\hline 2011-08-20 & 2361 & 313.69 & -585.72 & 52068.9 & -2.0 & 11273 & 661.35 & -601.9 & 1964.22 & - & - & - & $\mathrm{N}$ \\
\hline 2011-08-31 & 2413 & -311.4 & -428.77 & 27251 & 3.6 & 11281 & -455.58 & -420.77 & 8277.05 & 958.34 & 513.2 & C5.5 & $\mathrm{N}$ \\
\hline 2011-09-06 & 2704 & -549.72 & 54.52 & 33199.7 & -2.3 & 11288 & -460.72 & 221.59 & 830.88 & 1089.59 & -732.03 & - & $\mathrm{N}$ \\
\hline $2011-12-28$ & 3370 & -55.12 & -677.57 & 407905 & 1.0 & 11386 & -147.38 & -478.93 & 10610.8 & - & - & C4.0 & $\mathrm{Y}$ \\
\hline 2012-01-05 & 3472 & 160.58 & 0.15 & 24272.8 & -5.8 & 11392 & 291 & 391.97 & 733.79 & 1174.76 & -818.62 & $\mathrm{C} 2.2$ & $\mathrm{~N}$ \\
\hline 2012-01-12 & 3538 & -159.98 & 153.94 & 19884.5 & -4.9 & 11395 & 182.53 & 420.36 & 994.92 & 1489.52 & -592.93 & C1.6 & $\mathrm{Y}$ \\
\hline 2012-01-31 & 3701 & -191.75 & -53.83 & 53238.2 & -9.5 & 11413 & -108.41 & 235.74 & 467.9 & 1615.37 & -638.8 & - & $\mathrm{Y}$ \\
\hline $2012-02-15$ & 3839 & -58.66 & 69.83 & 34423.6 & -2.3 & 11417 & 143.7 & 630.68 & 1307.12 & - & - & - & Y \\
\hline 2012-03-11 & 4130 & -468.51 & 86.21 & 72066.4 & -1.8 & 11432 & -530.42 & 506.22 & 1329.08 & 1023.55 & -631.55 & C 3.8 & Y \\
\hline 2012-03-14 & 4130 & 183.02 & 78.97 & 87870.0 & -2.3 & 11432 & -231.64 & 357.68 & 4043.8 & 1406.28 & 716.95 & M2.8 & $\mathrm{Y}$ \\
\hline $2012-03-23$ & 4236 & -303.04 & 166.13 & 25672.3 & 2.1 & 11442 & -716.4 & 276.89 & 116 & 805.11 & 524.99 & - & $\mathrm{N}$ \\
\hline 2012-05-06 & 4548 & -267.31 & 518.12 & 49896.6 & -3.9 & 11474 & -350.07 & 344.71 & 870.72 & 1317.37 & -771.24 & - & $\mathrm{Y}$ \\
\hline 2012-06-02 & 4799 & -183.49 & 213.97 & 124054 & -3.6 & 11495 & 40.16 & -200.53 & 642.11 & 1508.16 & -868.74 & - & $\mathrm{Y}$ \\
\hline 2012-07-01 & 5062 & 232.1 & 102.47 & 82449.7 & -3.2 & 11513 & -41.34 & 193.07 & 3550.3 & 1191.5 & 492.01 & M2.8 & $\mathrm{Y}$ \\
\hline 2012-07-19 & 5332 & -262.47 & -480.52 & 38522.2 & 6.8 & 11525 & -456.4 & -369.11 & 8026.43 & 1261.5 & 459.23 & C1.5 & $\mathrm{Y}$ \\
\hline $2012-07-26$ & 5383 & 29.74 & -17.28 & 52382.4 & -2.3 & 115 & 78.75 & -391.71 & 5614.62 & 1330.25 & 854.84 & - & $\mathrm{N}$ \\
\hline $2012-08-21$ & 5799 & -329.92 & -57.72 & 45524.3 & -1.8 & 11548 & -589.74 & 243.9 & 6068.9 & 1232.33 & -534.86 & - & Y \\
\hline $2012-12-23$ & 7748 & -19.18 & 74.25 & 25091.7 & -3.7 & 11635 & -224.4 & 218.06 & 976.83 & 1438.98 & -474.98 & C1.9 & $\mathrm{Y}$ \\
\hline 2013-01-09 & 7960 & -33.51 & -575.41 & 77184.7 & 5.9 & & -326.83 & -476.05 & & 953.92 & -749.33 & - & $\mathrm{N}$ \\
\hline 2013-02-02 & 8302 & -183.27 & 523.62 & 99418.1 & -2.6 & 11665 & -325.12 & 250.64 & 3367.35 & 2018.12 & -596.31 & $\mathrm{C} 1.2$ & Y \\
\hline $2013-02-27$ & 8609 & 161.22 & 210.77 & 19404.9 & 2.0 & 11682 & 170.71 & -175.68 & 6352.27 & 1527.5 & -531.28 & - & $\mathrm{N}$ \\
\hline $2013-04-23$ & 9292 & 0.51 & 2.51 & 41871.9 & - & & -133.75 & 494.33 & 897.21 & 1351.94 & -524.11 & - & $\mathrm{N}$ \\
\hline $2013-07-03$ & 10361 & -106.38 & -494.94 & 17600.9 & 3.5 & 11786 & -116.39 & -527.02 & 1766.31 & 1032.98 & 709.99 & - & $\mathrm{N}$ \\
\hline 2013-07-17 & 10474 & -148.08 & 417.36 & 169913 & -2.9 & 11793 & -554.88 & 288.09 & 4259.57 & 844.03 & -395.71 & - & Y \\
\hline 2013-08-16 & 968 & -454.61 & -26.19 & 25500.1 & 4.0 & 11820 & -338.69 & -322.11 & 609.29 & - & - & - & $\mathrm{Y}$ \\
\hline 2013-08-19 & 10968 & 158.75 & -16.15 & 33455.4 & 5.3 & 11825 & 338.58 & 171.89 & 874.37 & 1604.6 & 859.09 & - & $\mathrm{Y}$ \\
\hline 2013-09-01 & 11240 & 1.24 & -195.43 & 34099.3 & -2.5 & & 369.43 & -291.29 & 1151.34 & 1427.72 & 553.67 & - & $\mathrm{Y}$ \\
\hline 2013-10-04 & 1791 & -621.88 & 278.85 & 37121.1 & -2.7 & 11856 & -700.63 & 65.78 & 1539.7 & 1068.7 & -381.28 & $\mathrm{C} 2.5$ & $\mathrm{~N}$ \\
\hline 2013-10-15 & 11961 & -352.61 & 506.78 & 58677.4 & -3.3 & 11868 & -552.44 & 221.7 & 304.36 & - & - & - & $\mathrm{N}$ \\
\hline $2013-12-12$ & 3120 & 129.13 & 577.67 & 180477 & -2.3 & & -192.19 & 291.67 & 1451.89 & 1269.92 & 853.51 & - & $\mathrm{N}$ \\
\hline $2013-12-28$ & 3441 & -488.18 & 76.52 & 59921.3 & 3.4 & 11937 & -712.35 & -184.64 & 1573.37 & 913.73 & -601.18 & - & $\mathrm{N}$ \\
\hline 2014-01-01 & 13441 & 286.95 & 181.73 & 66027.8 & 3.5 & 11937 & 54.52 & -207.13 & 398.35 & 920.68 & -557.42 & - & $\mathrm{Y}$ \\
\hline 2014-02-06 & 14086 & 21.99 & 490.11 & 108409 & -2.4 & 11972 & 306.93 & -99.95 & - & 1211.43 & 797.83 & C4.0 & $\mathrm{Y}$ \\
\hline $2014-02-23$ & 14314 & -272.94 & -93.04 & 33423.6 & -5.5 & 11988 & -433.16 & -69.32 & 636.68 & - & - & - & $\mathrm{N}$ \\
\hline 2014-03-03 & 14498 & -496.82 & 534.82 & 89720.2 & -2.7 & 11996 & -719.41 & 297.45 & 1090.75 & 781.35 & 570.78 & C1.8 & $\mathrm{N}$ \\
\hline 2014-03-20 & 14805 & 112.04 & 122.69 & 18424.9 & 6.2 & 12008 & -82 & -67 & - & - & - & - & $\mathrm{N}$ \\
\hline 2014-04-01 & 15094 & -304.2 & 556.84 & 41949.8 & -2.5 & 12022 & -347.26 & 355.14 & 1254.38 & 1401.98 & -578.53 & C 3.3 & $\mathrm{~N}$ \\
\hline 2014-04-24 & 15583 & 84.59 & -75.21 & 10143.5 & 11.2 & 12044 & 98.44 & -250.73 & 1168.44 & 1261.97 & -761.01 & - & $\mathrm{Y}$ \\
\hline 2014-07-30 & 17245 & -387.88 & -128.17 & 12974.8 & - & 12125 & -317.41 & -335.53 & 3070.08 & 1490.81 & 786.41 & C1.3 & $\mathrm{Y}$ \\
\hline 2014-08-10 & 17433 & 49.58 & 133.68 & 13631.8 & - & 12135 & -170.85 & 128.81 & - & 1949.58 & -541.86 & $\mathrm{C} 2.7$ & $\mathrm{Y}$ \\
\hline $2014-08-25$ & 17688 & -558.34 & -175.42 & 16473.2 & 22.3 & 12150 & -545.39 & -308.73 & - & 1488.22 & 451.37 & C1.0 & $\mathrm{N}$ \\
\hline 2014-09-22 & 18197 & -211.69 & -212.54 & 28449.2 & - & 12171 & -399.43 & -246.08 & - & 1457.12 & 735.74 & - & $\mathrm{Y}$ \\
\hline $2014-09-26$ & 18197 & 687.92 & -173.48 & 12332 & 6.9 & 12172 & 85.74 & -262.37 & - & 1581.69 & 786.71 & C8.7 & $\mathrm{N}$ \\
\hline 2014-10-17 & 18613 & -47.7 & 361.12 & 67726.7 & 1.0 & 12187 & -181.75 & -239.47 & 2785.12 & 2082.55 & 605.59 & - & $\mathrm{Y}$ \\
\hline 2014-11-16 & 18985 & -74.87 & -105.35 & 22287.4 & 18.6 & 12213 & -208.22 & -187.62 & - & 855.05 & -61.46 & - & $\mathrm{N}$ \\
\hline $2014-11-28$ & 19096 & 178.73 & 668.19 & 77996.8 & 0.9 & 12223 & 259.74 & 274.5 & 2438.98 & 1203.29 & 755.57 & - & Y \\
\hline $2014-12-12$ & 19275 & -215 & -21.93 & 18214 & 19.0 & 12232 & -197.91 & 175.04 & - & 1408.35 & -873.8 & - & Y \\
\hline 2014-12-17 & 19350 & -284.17 & 101.63 & 11277.4 & -20.5 & 12240 & -393.77 & 193.19 & - & 1597.41 & -568.17 & - & $\mathrm{Y}$ \\
\hline
\end{tabular}

